\title{
Laboreal
}

Volume 17 №2 | 2021

Trabalho e Território

\section{0 años de Médicos sin Fronteras y las reflexiones de la labor humanitaria contemporánea}

50 anos de Médicos sem Fronteiras e os reflexos do trabalho humanitário contemporâneo

Quand les 50 ans de Médecins sans Frontières rappelle les évolutions du travail humanitaire contemporain

50 years of Doctors without Borders and the reflections of contemporary humanitarian work

\section{Luciana B. Cavanellas}

\section{(2) OpenEdition}

\section{Journals}

Edición electrónica

URL: https://journals.openedition.org/laboreal/18220

DOI: $10.4000 /$ laboreal. 18220

ISSN: 1646-5237

\section{Editor}

Universidade do Porto

Referencia electrónica

Luciana B. Cavanellas, «50 años de Médicos sin Fronteras y las reflexiones de la labor humanitaria contemporánea», Laboreal [En línea], Volume 17 №2 | 2021, Publicado el 01 diciembre 2021, consultado el 05 diciembre 2021. URL: http://journals.openedition.org/laboreal/18220 ; DOI: https:// doi.org/10.4000/laboreal.18220

Este documento fue generado automáticamente el 5 diciembre 2021.

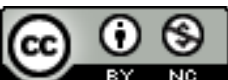

Laboreal está licenciado com uma Licença Creative Commons - Atribuição-NãoComercial 4.0 Internacional. 


\section{0 años de Médicos sin Fronteras y las reflexiones de la labor humanitaria contemporánea}

50 anos de Médicos sem Fronteiras e os reflexos do trabalho humanitário contemporâneo

Quand les 50 ans de Médecins sans Frontières rappelle les évolutions du travail humanitaire contemporain

50 years of Doctors without Borders and the reflections of contemporary

humanitarian work

Luciana B. Cavanellas

\section{NOTA DEL EDITOR}

Traducido por :

Fernanda Romero (fernandaromero.trad@gmail.com)

Manuscrito recibido en : 19/09/2021

Aceptado tras peritaje : 07/10/2021

"La presencia de MSF aquí es como un rayo de luz en la borrasca" (Dunne, 2012, p. 101)

\section{El nacimiento de MSF}

1 La historia del movimiento humanitario contemporáneo tiene como hitos importantes la creación de la Cruz Roja (1863) y de la Convención de Ginebra, en 1864, firmada por 12 potencias europeas, inaugurando al llamado derecho comunitario internacional. 
2 La iniciativa es del filántropo suizo Henri Dunant, cuyo libro "Un Souvenir de Solferino" (1862), basado en las atrocidades de la guerra da Lombardía y en la agonía de miles de heridos en el campo de batalla, propone la creación de sociedades permanentes de socorro a los heridos (Brauman, 2009).

3 Más de cien años después, en 1986, un anuncio de la Cruz Roja que decía "Se buscan médicos para Biafra" atrae a jóvenes médicos franceses y otorga un nuevo impulso a los movimientos de solidaridad internacional (Aubrée, 2004).

4 La guerra de Biafra, iniciada en 1967, cuando declaró su independencia de Nigeria, provocó una reacción militar del gobierno nigeriano que, además de miles de muertos, colocó a unos 8 millones de biafreños en una situación de hambruna devastadora. Según Ferré (2007), las imágenes de la primera hambruna televisada conmocionaron al mundo y pusieron de manifiesto la impotencia de instituciones clásicas como la ONU y la Cruz Roja.

5 Entre los médicos que se ofrecieron como voluntarios para la misión en Biafra, siguiendo los principios de neutralidad de la Cruz Roja, estaba Bernard Kouchner, de 32 años, quien, inconformado con los horrores de que había sido testigo, decidió romper el silencio y, junto con otros médicos voluntarios y periodistas, creó Médicos Sin Fronteras (MSF) en Francia en 1971. Con el compromiso de aunar la ayuda médica y el testimonio, en favor de las poblaciones en peligro, surgió con MSF una "era de médicos franceses que se marchan para atender más allá de las fronteras y de las separaciones ideológicas" (Aubrée, 2004, p. 13). Denominados "sin fronteras", estos médicos, a diferencia de otras ONG en las que actuaban con discreción, pretenden actuar como médicos y también como testigos. "Ellos ayudan, cuidan e informan". Dondequiera que haya sufrimiento humano, la prioridad es "actuar y actuar rápido" (Ferré, 2007, p. 21).

En diciembre de 1971, la revista médica Tonus anunció el nacimiento de MSF en una convocatoria a los médicos:

"(...) votaremos a favor de esta movilización de deseos decididos a derribar todas las barreras, todas las fronteras que todavía existen entre los que tienen vocación de salvar, de sangrar, y las víctimas de la barbarie humana o de las perturbaciones de la naturaleza, de las olas gigantescas, de los terremotos y de otras catástrofes que hunden en el luto a los países que a menudo están menos preparados para hacerles frente" (Ferré, 2007, p. 21, traducción libre).

7 Dispuesto a romper las fronteras entre los que tienen condiciones de ayudar y aquellos que necesitan ayuda, el movimiento humanitario se enfrentará a una serie de conflictos y desafíos de la más diversa índole, que le harán enfrentarse, en ocasiones, a sus propias convicciones.

\section{Expandiendo fronteras}

8 En 1978, la operación Un bateau pour le Vietnam, apoyada por iniciativa de intelectuales como Jean Paul Sartre y Raymond Aron y organizada por Bernard Kouchner (Ferré, 2007), con el objetivo de salvar personas en el mar de China, llamó la atención de la opinión pública para los miles de personas que huían de sus países en embarcaciones improvisadas. 
9 Esta operación fue desaprobada por MSF, lo que culminó con la salida de Kouchner, que más tarde vino a fundar la ONG Médicos del Mundo (MDM). En la opinión de Brauman (2003), esta escisión reflejó un momento en el que MSF empezó a expresar la necesidad de estructurarse mejor. Ante el enfrentamiento de situaciones inéditas, han surgido dos corrientes distintas en el seno de MSF: una que se remonta a los orígenes, cuando los profesionales salían al terreno con su "maleta de emergencia" y se dedicaban a movilizar a la opinión pública y a las organizaciones con mayor poder de intervención. Otra, para quienes esas misiones ya no bastaban y se hacía necesario crear una estructura de actuación propia e independiente. En 1979, los primeros, los legitimistas, fueron derrotados por los llamados independentistas.

El movimiento denominado French Doctors dominó la escena humanitaria de los años ochenta, suscitando vocaciones e influyendo en el surgimiento de una serie de asociaciones especializadas en emergencias, como: ACF (Action contre la faim), ÉQUILIBRE, HI (Handicap International) o AMI (Aide médicale internationale). Privilegiando la asistencia, pero también basadas en la defensa de los derechos humanos, estas organizaciones han utilizado sistemáticamente los medios de comunicación social para dar a conocer los dramas humanos en los lugares más lejanos (Ferré, 2007).

\section{La afirmación del "espacio humanitario"}

11 La hambruna en Etiopía se convirtió en uno de estos acontecimientos emblemáticos, cuando en 1984 un periodista fue autorizado por las autoridades etíopes a filmar el drama, emitido por la $\mathrm{BBC}$, que conmocionó al mundo. El efecto de esta emisión generó uno de los mayores movimientos de solidaridad en la opinión pública mundial. Se envió una gran cantidad de géneros alimenticios a Etiopía, pero las autoridades locales comenzaron a desviar parte del cargamento, poniéndolo al servicio de su política de desplazamiento de la población (Ferré, 2007). Esto ha provocado cuestionamientos y reflexiones necesarios al sector humanitario.

12 En este mismo contexto, también en Etiopia, ocurrió el primer enfrentamiento entre una organización humanitaria y el Estado en cuyo territorio estaba trabajando. Según Brauman (2003), al protestar contra la utilización de medios logísticos procedentes de la ayuda internacional para el desplazamiento forzado de la población, MSF fue expulsada del país, ante el silencio de las Naciones Unidas y de otras ONG que dependían de financiación.

13 La situación de la manipulación de los medios de ayuda en Etiopía ha puesto de manifiesto lo inestable que puede ser el ámbito humanitario, ya que, en la mayor parte de las veces, se encuentra inmerso en un complejo contexto político. Por tratarse de un trabajo que tiene lugar en situaciones de crisis y sufrimiento, además de la movilización de ayuda y tratamiento, son muchos los dilemas que acechan a la acción humanitaria, que se enfrenta a difíciles decisiones en un debate constante sobre normas y valores (Schwartz \& Durrive, 2007).

14 La ayuda no siempre es posible porque implicaría el silencio ante las atrocidades presenciadas. Cuando la denuncia y la atención a las víctimas no pueden conciliarse, ¿qué hay que hacer? ¿Cómo mantener los principios humanitarios ante ciertas negociaciones difíciles y necesarias con las autoridades locales? ¿Cómo mantener la 
credibilidad en sus acciones e intenciones cuando los gobiernos utilizan la imagen construida por el movimiento humanitario para servir a sus intereses políticos y económicos? Para Ferré (2007), las fronteras, ya permeables, entre política y humanitarismo se han difuminado definitivamente y las misiones de las ONG son cada vez más delicadas, ya que se desarrollan necesariamente en el corazón de la política.

En 1995, la Cruz Roja, junto con otras importantes organizaciones, reafirmó el derecho internacional humanitario y el principio de imparcialidad, según el cual la asistencia debe obedecer a la necesidad de aliviar el sufrimiento de las personas. En 2007, el Consenso Europeo sobre Ayuda Humanitaria invitó a las organizaciones a implementar sus propias políticas, independientes de las políticas gubernamentales, respetando los principios de "humanidad, neutralidad, imparcialidad e independencia". Surge la idea de "espacio humanitario", con la independencia como uno de sus fundamentos (Coconnier \& Pommier, 2009). A estos cuatro principios, la ONG Médicos Sin Fronteras añade la Transparencia y la Ética Médica, reforzando su compromiso con sus donantes y su papel de prestar asistencia médica por encima de todo.

\section{Los riesgos de la motivación humanitaria}

16 Sin embargo, a pesar de la constante reafirmación de sus principios, la dialéctica entre seguridad e independencia se mantiene y se ha hecho más compleja a lo largo de los años en el interior del movimiento humanitario. Si antes, en torno a los años 80 y 90 , los logotipos, los coches y las banderas de las ONG significaban una garantía de respeto en el terreno, hoy en día, a menudo optan por un cierto anonimato e invisibilidad, considerando que los mandatos y las banderas ya no garantizan la protección de los trabajadores (Coconnier \& Pommier, 2009).

Entre 2003 y 2007, 88 trabajadores murieron en misión, siendo el personal local el más afectado (Coconnier \& Pommier, 2009). El año 2006 fue considerado uno de los más violentos, con constantes ataques y el asesinato de 17 profesionales de la ONG Acción contra el Hambre en Sri Lanka. Según una investigación publicada en aquel entonces, las estadísticas mostraron que en 10 años, desde 1997, 408 actos de violencia distintos se cobraron 947 víctimas, de las cuales 434 muertos. Una vez más, los trabajadores locales fueron los más afectados (Brauman, 2009). Para mencionar un hecho más reciente relatado en la web oficial de MSF [1], en 2020, la maternidad de MSF en Kabul sufrió un brutal ataque a tiros en el que murieron 16 madres, 2 niños y 1 obstetra de MSF.

18 A pesar de ello y de lo que las circunstancias en contextos violentos nos llevan a imaginar, la "profesión" humanitaria no se considera estadísticamente más peligrosa en comparación con muchas otras (Brauman, 2009). En la misma línea, la exposición al riesgo no parece representar una dificultad significativamente mayor a la hora de contratar o incluso de retener a los trabajadores en el ámbito humanitario. Marcado por una alta tasa de rotación de sus profesionales en las ONG internacionales, la experiencia de actuar en contextos de alto riesgo por sí sola no resultó relevante en la decisión de aceptar otro destino (Korff, Balbo, Mills, Heyse, \& Wittek, 2015). Como ya hemos subrayado, "los trabajadores de MSF tienen la libertad de elegir si aceptan o no trabajar en misiones que impliquen mayores riesgos. $Y$ una vez que aceptan, reciben todas las instrucciones necesarias para minimizarlos; las reglas de la organización tienen como objetivo proteger a los profesionales que han aceptado exponerse" (Cavanellas \& Brito, 2019, p. 15). 
19 Movidos por un sentimiento de justicia, compasión y solidaridad, ingresar en una ONG como MSF puede significar la realización de un sueño y la confirmación de una motivación humanitaria, considerada fundamental para afrontar las adversidades en la misión.

\section{El campo macro político y la experiencia en el terreno}

20 Sin embargo, muchos son los dilemas que se plantean en el ámbito humanitario a nivel macro, que implican decisiones importantes sobre las negociaciones políticas, la capacidad de gestión de conflictos, la movilización de recursos humanos y técnicos y la conservación de la imagen. Hay todavía que considerar cuestiones más delicadas como el derecho de injerencia o el derecho a intervenir en otro Estado; la sombra que proyecta la medicina colonial y el neocolonialismo europeo sobre los países del Tercer Mundo; la utilización del legado (patrimonio adquirido) en favor de intereses turbios y la ayuda que se convierte en industria. Dificultades concretas y dilemas éticos relacionados con el campo de la ayuda internacional.

21 Pero "la responsabilidad se construye en la experiencia y en el encuentro de los sujetos concretos, en la experiencia directa de las situaciones vividas por esos sujetos, haciendo de la figura de la vulnerabilidad el lugar para la incorporación de una ética de la responsabilidad" (Saillant, 2010, p. 63). Es en el cuerpo a cuerpo donde tiene lugar la actividad humana. Amparados por las estructuras de las organizaciones, los trabajadores llevan a cabo en el terreno, con los pies en la tierra, su acción y su misión. Se ven afectados y transformados por las circunstancias, y también afectan y transforman a las personas y los lugares por los que pasan.

Los retos del "sin fronteras" se viven en el ámbito de las relaciones que se establecen en el terreno, donde hay que dejarse penetrar por la cultura local, conociendo e intentando comprender los códigos y valores, dialogando con lo verdaderamente extranjero. Atender a los extranjeros y trabajar con el personal local fomenta la exposición a conexiones intersubjetivas inéditas, lo que requiere que el expatriado sea flexible y esté abierto al diálogo.

En la actuación humanitaria, es necesario sustituir la bata blanca por las ropas de un ciudadano común (Brauman, 2003). Después de todo, sólo en el diálogo, donde lo diferente tiene voz, la compasión puede dar lugar a la solidaridad (Caponi, 2000). A consecuencia de la importancia de las experiencias de los profesionales en la construcción de la identidad del trabajo humanitario, cada vez más expuestas en diarios y blogs, es importante valorar no sólo las macro, sino también las micro intervenciones en el campo, desplazando el enfoque de la atención y del interés masivamente centrado en las instituciones hacia los individuos. En opinión de investigadores como Fechter (2012), las relaciones y los valores personales contribuyen significativamente a las perspectivas del trabajo humanitario, y es crucial reconocer a los trabajadores como agentes de cambio, que viven en medio de una serie de contradicciones entre la vida personal y la profesional.

La necesidad de construir una carrera más sólida o una vida afectivo-sexual más estable, además del desgaste físico-emocional que impone el trabajo en el terreno, representan buena parte de las exigencias personales en el trabajo humanitario 
(Cavanellas \& Brito, 2019), siendo fundamental que el compromiso en el cuidado del otro se complemente con el compromiso en el cuidado de sí mismo (Fechter, 2012). No obstante la mística altruista y el heroísmo asociados a la acción humanitaria, la actividad se desarrolla en el mundo real del trabajo (Lancman \& Sznelwar, 2008) y se basa efectivamente en el propósito de socorrer, en momentos críticos, a seres humanos, apoyándolos y ayudándolos a recuperarse para que puedan volver a su camino (Brauman, 2003).

\section{La fuerza de la solidaridad}

Con oficinas en 28 países y actuación en más de 70, Médicos Sin Fronteras representa una capacidad de prestar asistencia sanitaria a escala internacional gracias a las operaciones colaborativas entre sus diferentes asociaciones. Con más de $45 \mathrm{mil}$ profesionales de distintas áreas y nacionalidades, entre expatriados y personal local, y con el $96 \%$ de su financiación procedente de donaciones individuales y de la iniciativa privada, MSF cumple 50 años con un Premio Nobel de la Paz en su historia y como uno de los símbolos más elocuentes del movimiento humanitario contemporáneo.

\section{BIBLIOGRAFÍA}

Aubrée, C. (2004). Les métiers de l’humanitaire et de la solidarité. Paris: L'Etudiant.

Brauman, R. (2003). Humanitario el dilema. Barcelona: Icaria.

Brauman, R. (2009). La Médecine humanitaire. Paris: PUF.

Caponi, S. (2000). Da compaixão à solidariedade. Rio de Janeiro: Editora Fiocruz.

Cavanellas, L. B., \& Brito, J. (2019). Os desafios do cuidado em situações-limite: as dramáticas da atividade no trabalho humanitário. Laboreal, 15(2), 1-26. https://doi.org/10.4000/laboreal.14966

Coconnier, M-L., \& Pommier, B. (2009). L'Action Humanitaire. Paris: PUF.

Lancman, S., \& Sznelwar, L. (2008). Christophe Dejours: da psicopatologia à psicodinâmica do trabalho. Rio de Janeiro: Ed. Fiocruz.

Dunne, C. (2012). Khayelitsha, cidade do Cabo. In M. Llosa, E. Brum, P. Giordano, C. Dunne, A. Barlett, J. Levine, E. Aykel, T. Doshi, \& W. N`Sondé (Eds.), Dignidade! (pp. 71-114). São Paulo: Leya.

Fechter, A-M. (2012). The Personal and the Professional: Aid workers' relationships and values in the development process. Third World Quarterly, 33(8), 1387-1404. https://doi.org/ 10.1080/01436597.2012.698104

Ferré, J-L. (2007). L'action humanitaire. Toulouse: Milan.

Korff, V., Balbo, N., Mills, M., Heyse, L., \& Wittek, R. (2015). The impact of humanitarian context conditions and individual characteristics on aid worker retention. Disasters, 39(3), 522-545.

https://doi.org/10.1111/disa.12119 
Saillant, F. (2010). A Responsabilidade na intervenção humanitária: indiferença ou engajamento? In J. Ferreira, \& P. Schuch (Orgs.), Direitos e ajuda humanitária: perspectivas sobre família, gênero e saúde (pp. 49-75). Rio de Janeiro: Editora Fiocruz.

Schwartz, Y., \& Durrive, L. (2007). Trabalho e Ergologia, conversas sobre a atividade humana. Niterói: EDUFF.

\section{NOTAS}

1. Recogido en 6 de septiembre de 2021 de https://www.msf.org.br/nossa-historia

\section{RESÚMENES}

La ONG internacional Médicos Sin Fronteras (MSF) cumple 50 años en diciembre de 2021. Su historia y sus acciones revelan acontecimientos importantes que son necesarios para comprender el movimiento humanitario contemporáneo. Desde las negociaciones en contextos políticos complejos hasta las experiencias personales de los trabajadores que se arriesgan para prestar ayuda y solidaridad, los conflictos y dilemas son constantes cuando se trata de conciliar la medicina y ser testigo del sufrimiento humano. Para fundamentar y enriquecer esta reflexión, este texto pretende presentar las aportaciones de los estudiosos y protagonistas de la labor humanitária, dispuestos a mostrar las dificultades a las que se enfrentan y las contradicciones que hay que superar. Con más de 45 mil profesionales, trabajando en más de 70 países, MSF sigue preservando sus principios, desafiando las fronteras y tratando de llevar atención a los necesitados.

A ONG internacional Médicos sem Fronteiras (MSF) faz 50 anos em dezembro de 2021. A sua história e ação revelam eventos importantes e necessários à compreensão do movimento humanitário contemporâneo. Das negociações em contextos políticos complexos às vivências pessoais de trabalhadores que se arriscam para prestar ajuda e solidariedade, os conflitos e dilemas são constantes quando se trata de conciliar medicina e testemunho do sofrimento humano. Para fundamentar e enriquecer a reflexão, este texto procura apresentar contribuições de estudiosos e protagonistas do trabalho humanitário, dispostos a mostrar as dificuldades enfrentadas e as contradições a serem superadas. Com mais de 45 mil profissionais, atuando em mais de 70 países, MSF segue preservando seus princípios, desafiando fronteiras e buscando levar cuidado a quem precisa.

L'ONG internationale Médecins Sans Frontières (MSF) complète 50 ans en décembre 2021. Son histoire et ses actions révèlent des événements importants qui sont nécessaires pour comprendre le mouvement humanitaire contemporain. Qu'il s'agisse de négociations dans des contextes politiques complexes ou de l'expérience personnelle des travailleurs qui prennent des risques pour apporter aide et solidarité, les conflits et les dilemmes sont constants lorsqu'il s'agit de concilier médecine et témoignage de la souffrance humaine. Pour fonder et enrichir cette réflexion, ce texte cherche à présenter des contributions de chercheurs et de protagonistes du travail humanitaire, désireux de montrer les difficultés rencontrées et les contradictions à surmonter. Avec plus de 45.000 professionnels, travaillant dans plus de 70 pays, MSF continue à 
préserver ses principes, à défier les frontières et à chercher à apporter des soins à ceux qui en ont besoin.

The international NGO Doctors Without Borders (DWB) turns 50 years old in December 2021. Its history and actions reveal important events that are necessary to understand the contemporary humanitarian movement. From negotiations in complex political contexts to the personal experiences of workers who risk themselves to provide aid and solidarity, the conflicts and dilemmas are constant when it comes to reconciling medicine and witnessing human suffering. To ground and enrich the reflection, this text seeks to present contributions from scholars and protagonists of humanitarian work, willing to show the difficulties faced and the contradictions to be overcome. With more than 45 thousand professionals, working in over 70 countries, MSF continues to preserve its principles, challenging borders and seeking to bring care to those in need.

\section{ÍNDICE}

Keywords: humanitarian movement, doctors without borders, care, witness, solidarity Palavras-chave: movimento humanitário, médicos sem fronteiras, cuidado, testemunho, solidariedade

Palabras claves: movimiento humanitario, médicos sin fronteras, atención, testigo, solidaridad Mots-clés: mouvement humanitaire, médecins sans frontières, soins, témoignage, solidarité

\section{AUTOR}

\section{LUCIANA B. CAVANELLAS}

https://orcid.org/0000-0003-0562-6507

Fundação Oswaldo Cruz, Coordenação de Saúde do Trabalhador (CST/Cogepe/Fiocruz), Avenida Brasil, 4365. Pavilhão Carlos Augusto da Silva. Manguinhos, Rio de Janeiro, Brasil, 21040-36

luciana.cavanellas@fiocruz.br 